\title{
Unsteady thick airfoil aerodynamics: experiments, computation, and theory
}

\author{
C. Strangfeld* \\ Institute of Fluid Dynamics and Technical Acoustics, \\ Technische Universität Berlin, 10623 Berlin, Germany \\ C. L. Rumsey ${ }^{\dagger}$ \\ NASA Langley Research Center, Hampton, Virginia 23681 \\ H. Müller-Vahl ${ }^{\ddagger}$ and D. Greenblatt $\S$ \\ Technion - Israel Institute of Technology, 32000 Haifa, Israel \\ C. N. Nayeri" and C. O. Paschereit " \\ Institute of Fluid Dynamics and Technical Acoustics, \\ Technische Universität Berlin, 10623 Berlin, Germany
}

\begin{abstract}
An experimental, computational and theoretical investigation was carried out to study the aerodynamic loads acting on a relatively thick NACA 0018 airfoil when subjected to pitching and surging, individually and synchronously. Both pre-stall and post-stall angles of attack were considered. Experiments were carried out in a dedicated unsteady wind tunnel, with large surge amplitudes, and airfoil loads were estimated by means of unsteady surface mounted pressure measurements. Theoretical predictions were based on Theodorsen's and Isaacs' results as well as on the relatively recent generalizations of van der Wall. Both two- and three-dimensional computations were performed on structured grids employing unsteady Reynolds-averaged Navier-Stokes (URANS). For pure surging at pre-stall angles of attack, the correspondence between experiments and theory was satisfactory; this served as a validation of Isaacs theory. Discrepancies were traced to dynamic trailing-edge separation, even at low angles of attack. Excellent correspondence was found between experiments and theory for airfoil pitching as well as combined pitching and surging; the latter appears to be the first clear validation of van der Wall's theoretical results. Although qualitatively similar to experiment at low angles of attack, two-dimensional URANS computations yielded notable errors in the unsteady load effects of pitching, surging and their synchronous combination. The main reason is believed to be that the URANS equations do not resolve wake vorticity (explicitly modeled in the theory) or the resulting rolled-up unsteady flow structures because high values of eddy viscosity tend to "smear" the wake. At post-stall angles, three-dimensional computations illustrated the importance of modeling the tunnel side walls.
\end{abstract}

${ }^{*} \mathrm{PhD}$ student, Hermann-Föttinger Institut, ISTA, Technische Universität Berlin, Müller-Breslau-Str. 8, 10623 Berlin, strangfeld@tu-berlin.de

${ }^{\dagger}$ NASA Senior Researcher, Computational AeroSciences Branch, Fellow AIAA

${ }^{\ddagger}$ PhD student, Faculty of Mechanical Engineering, Technion, Haifa 32000, Israel, hannsmv@technion.ac.il

$\S$ Associate Professor, Faculty of Mechanical Engineering, Technion, Haifa 32000, Israel, Associate Fellow AIAA

『Research Assistant, Hermann-Föttinger Institut, ISTA, Technische Universität Berlin, Müller-Breslau-Str. 8, 10623 Berlin

" Professor, Hermann-Föttinger Institut, ISTA, Technische Universität Berlin, Müller-Breslau-Str. 8, 10623 Berlin 


\section{Nomenclature}

\begin{tabular}{|c|c|c|c|}
\hline$\alpha$ & Angle of attack, ${ }^{\circ}$ & $\beta_{0}$ & Angle of attack oscillation amplitude, ${ }^{\circ}$ \\
\hline$\gamma$ & Vortex sheet strength, $\mathrm{m} / \mathrm{s}$ & $\lambda$ & Wave length of the oscillating, $\mathrm{m}$ \\
\hline$\rho$ & Air density, $\mathrm{kg} / \mathrm{m}^{3}$ & $\sigma$ & $\begin{array}{l}\text { Relative velocity amplitude of the oscillating } \\
\text { free stream }\end{array}$ \\
\hline$\phi$ & Phase angle, ${ }^{\circ}$ & $\omega$ & Angular frequency, $1 / \mathrm{s}$ \\
\hline$\omega_{s}$ & Rotational speed, rad/s & $\Gamma$ & Circulation, $\mathrm{m}^{2} / \mathrm{s}$ \\
\hline$a$ & Non-dimensional pitch axis & $c$ & Airfoil chord length, m \\
\hline$k$ & Reduced frequency, $\omega c /\left(2 u_{s}\right)$ & $l$ & Coefficients from Isaacs \\
\hline$m$ & Wave number & $p$ & Static pressure, $\mathrm{Pa}$ \\
\hline$t$ & Airfoil thickness, m & $u$ & Free stream velocity, $\mathrm{m} / \mathrm{s}$ \\
\hline$C_{l}$ & Lift coefficient & $C_{p}$ & Pressure coefficient \\
\hline$C(k)$ & Theodorsen function & $F(k)$ & Real part of the Theodorsen function \\
\hline$G(k)$ & Imaginary part of the Theodorsen function & $H$ & Coefficient from van der Wall \\
\hline$J$ & Bessel function of the first kind & $L$ & Lift, $\mathrm{N}$ \\
\hline$R$ & Radius of a vertical axis wind turbine, $\mathrm{m}$ & $R e$ & Reynolds number \\
\hline \multicolumn{4}{|c|}{ Subscript } \\
\hline$q s$ & Quasi steady & ref & Reference \\
\hline$s$ & Steady & st & static \\
\hline us & Per unit span & & \\
\hline \multicolumn{4}{|c|}{ Operators } \\
\hline$\overline{()}$ & Time averaged & \langle\rangle & Phase averaged signal \\
\hline 1 & Turbulence of a signal & $\Delta$ & Difference \\
\hline
\end{tabular}

\section{Introduction}

During the first half of the 20th century, the study of unsteady aerodynamics was motivated by problems associated with wing flutter, the estimation of helicopter blade loads, and the effect of wind gusts on aircraft. Many of these problems remain relevant today. In recent years, the stability and survivability of small low flying air vehicles that are exposed to highly unsteady winds have produced renewed interest in the topic. Furthermore, the desire for robust design of wind turbines - whose blades are exposed to highly unsteady flows produced by, inter alia, yaw misalignments and atmospheric turbulence - requires an urgent need for the accurate prediction of unsteady loads. ${ }^{1}$ In this paper, two sources of unsteadiness are explored. On the one hand, the attached flow in unsteady conditions like an oscillating free stream or a pitching airfoil at low angles of attack is studied. Although the flow remains fully attached, significant unsteady lift overshoot has been documented, see Refs. 2-4. On the other hand, the phenomenon of dynamic stall is investigated, which is characterized by the shedding of a strong vortex across the suction surface that induces rapid load excursions. Dynamic stall itself is already a highly unsteady phenomenon; nevertheless, combined studies including an oscillating free stream are rare and a lack of validation still exists.

Considering the fully attached airfoil, theoretical approaches to unsteady aerodynamics commenced about two decades after the work of Kutta ${ }^{5}$ and Joukowski (Zuhkouski). ${ }^{6}$ Calculations of forces acting on a flat plate in unsteady plunge motion are published in Wagner. ${ }^{7}$ It also provided a first approach to calculate the lift of airfoils in pitching motion, which is the basis for the development of a complete theory for pitching motion in Glauert. ${ }^{8}$ Challenged by the problem of wing flutter, a landmark innovation occurred with the development of a general analytical solution for airfoils undergoing angle of attack oscillations and plunge motion in Theodorsen. ${ }^{2}$ All velocity potentials were calculated for a steady stream potential flow and the unsteady circulation was computed, where the wake vorticity is determined by the Kutta condition. Today, we refer to the Theodorsen function that includes multiples of the reduced frequency as its argument.

The need for more accurate estimations of helicopter blade loads motivated Isaacs ${ }^{3}$ to extend Theodorsen's work to include an oscillating free stream with no phase lag between the leading and trailing edges. A year later, the theory was extended to include angle of attack oscillations at the same frequency. ${ }^{9}$ Greenberg ${ }^{10}$ 
developed a solution for the dynamic lift and moment of a flat plate in an oscillating free stream undergoing angle of attack oscillations and plunge motion. Furthermore, this approach included an arbitrary phase lag between the oscillating free stream and the periodically pitching wing. However, this modulation of the unsteady free stream also implied a fore-aft wing motion. More than 30 years later, a new theory was developed, which was directly motivated by helicopter aerodynamics. Its focus was the prediction of the dynamic drag behavior under unsteady free stream. The derivation was based on an order of magnitude approximation to the in-plane perturbation velocity. ${ }^{11}$ Its author asserted that this assumption is more plausible than the high frequency assumption in Ref. 10. However, this theory was limited to small free stream fluctuations. In 1991, an extensive review of existing theoretical approaches was provided and solutions for harmonic plunge motion and unsteady angle of attack variations including arbitrary multiples of the free stream harmonic were provided, see Refs. 4,12-14. The review concluded that Isaacs' theory is the only "exact theory" without additional simplifications. Furthermore, significant deviations of Greenberg's theory for relative velocity amplitudes higher than $\sigma=0.4$ were determined. Based on Theodorsen's approach, an analytical framework was developed for variable geometry airfoils including camberline elasticity in arbitrary motion. However, this ansatz did not consider the effect of an unsteady free stream, see Ref. 15.

In addition to fully attached flow configurations, detached flow phenomena are investigated in this study as well. Dynamic stall is a fascinating fluid dynamics phenomenon observed on rotorcraft and wind turbines when their blades pitch rapidly beyond the static stall angle, $\alpha_{s t}$. Excellent reviews of dynamic stall and its prediction have been published. ${ }^{16,17}$ Carr $^{16}$ describes approximately eleven stages of airfoil dynamic stall at high Reynolds numbers $R e>10^{6}$. In summary: reversed flow initially appears on the airfoil upper surface and spreads; the dynamic stall vortex (DSV) forms near the leading edge producing increases in lift and moment; finally, the vortex sheds from the airfoil leading to lift stall. Ekaterinaris et al. ${ }^{17}$ also provided a summary of many CFD efforts to predict dynamic stall, and describe important issues related to turbulence and transition modeling when using unsteady Reynolds averaged Navier-Stokes (URANS) methodologies. Many other CFD studies for dynamic stall have been conducted since the time of the review, including flow control, low Reynolds number studies, and three-dimensional applications (e.g., Refs. 18-23).

On wind turbines, dynamic stall manifests in different ways depending upon the type of turbine. On vertical axis wind turbines (VAWTs), blades stall dynamically at low blade tip speed to wind speed ratios, $\lambda=\omega_{s} R / u_{s}$ ( $\omega_{s}$ is the rotational speed, $R$ is the turbine radius and $u_{s}$ is the wind speed), resulting in loss of power and unsteady loads. Understanding and modeling of dynamic stall, particularly due to the current renewed interest in VAWT power, is of major scientific and technological importance (e.g., Refs. 24,25 and many others). For structural reasons, these blades are usually thick $(t / c \approx 18 \%)$ and the Reynolds numbers can be low, typically between $5 \cdot 10^{4} \leq R e \leq 10^{6}$, particularly on smaller urban and off-grid machines.

Experiments involving an unsteady free stream have been achieved using many novel designs (see Refs. 2630 and the references therein). In experiments involving stalled flows, some remarkable results have been obtained when the free stream varies according to $u(t)=u_{s}(1+\sigma \cos (\omega t))$, where $\sigma$ is the relative velocity oscillation amplitude, and the angular frequency, $\omega=2 \pi f$. For example, Gursul and $\mathrm{Ho}^{31}$ measured mean lift coefficients on a NACA 0012 airfoil at $\alpha=20^{\circ}$ far in excess of comparable quasi static values. The lift depended strongly on velocity oscillation amplitude (also see Ref. 32) and frequency, producing instantaneous lift coefficient values that were, amazingly, larger than 10 , with optimum reduced frequencies $k=\omega c / 2 u_{s} \approx$ $\mathcal{O}(1)$. The source of the high lift is a large vortex that is momentarily trapped on the airfoil and then swept downstream. Similar observations were made on delta wings at high angles of attack (Refs. 33-36). The only investigation of two-dimensional dynamic stall combined with an unsteady free stream appears to be that of Pierce et al. ${ }^{37}$ Although their angle of attack range was small $\alpha(t)= \pm 4^{\circ}$, the authors observed a large variation in the post-stall pitching moment coefficient as a result of the flow unsteadiness.

As discussed above, fully attached flow in unsteady conditions yield significant lift overshoots. Thus, it is surprising that many of these theories, in particular those of Greenberg and Isaacs, have not been fully validated experimentally or numerically, especially for large free stream oscillation amplitudes. ${ }^{38}$ Favier et al. ${ }^{39,40}$ investigated a pitching NACA 0012 airfoil in unsteady free stream. The wind tunnel generated high reduced frequencies up to $k=1.6$ and relative velocity amplitudes of more than $\sigma=0.35$. Although the airfoil lift shows significant dynamic effects, a comparison to Isaacs' theory was not considered. More recently, Granlund et al. ${ }^{41}$ investigated a NACA 0009 experimentally over a broad range of reduced frequencies at small relative velocity amplitude of $\sigma=0.1$. The reason for this lack of validation is unclear, but apparently the existing experimental facilities lack the large amplitude unsteady parameter range. Various wind tunnel challenges include fan stall, and large lag effects due to inertia and acoustic resonance. Indeed, a review 
of facilities reveals that the vast majority of them simulate only changes in airfoil angle of attack, namely pitching or plunging. ${ }^{42-44}$ Tunnels that produce an unsteady free stream are rare. The most common approach is to modify a standard steady wind tunnel to produce unsteady flows, ${ }^{37,45-47}$ typically using rotating vanes, louvers or some variation of this idea. Some tunnels combine the independent capabilities of angle of attack and wind speed variation. ${ }^{39,48,49}$ An exception is the approach of Favier et al., ${ }^{40}$ which employs a two degrees of freedom construction to pitch and translate the airfoil. Recently, an unsteady wind tunnel was developed to produce large amplitude oscillations of the free stream in Furman et al. ${ }^{50}$ Problems of fan stall, large inertial effects, and acoustic resonance were overcome during the initial design and testing phases. The tunnel proved to be ideally suited to validate large amplitude unsteady effects and, in particular, to assess the validity of theoretical approaches.

On helicopter blades, on VAWTs, and on HAWTs exposed to yaw misalignment, dynamic stall is accompanied by other sources of unsteadiness. In particular, the physical reality involves a dynamic change in pitch angle with a simultaneous change in flow speed. However, this simultaneous variation/oscillation is almost never reproduced experimentally in the context of airfoil dynamic stall. In the field of VAWTs, a notable exception is the study by Ferreira et al., ${ }^{24}$ who made two-dimensional particle image velocimetry (PIV) measurements on a small VAWT at $R e<10^{5}$ and tracked the dynamic stall vortex at different phase angles, $\phi=\omega t$. However, we have chosen a NACA 0018 airfoil as the focus of our study. ${ }^{51}$ Most of the static testing of NACA 0018 airfoils has been conducted at high Reynolds numbers appropriate to the aircraft industry and large turbines. ${ }^{52,53}$ Less research has been conducted at $R e<10^{6}$. Aerodynamic performance is significantly affected as the Reynolds number decreases below $R e=3 \cdot 10^{5}$ as shown in the investigations of Jacobs and Sherman ${ }^{54}$ and Raghunathan and Ombaka, ${ }^{55}$ and more recently in Timmer ${ }^{56}$ and Gerakopulos et al. ${ }^{57}$ Similarly, dynamic stall on NACA 0018 airfoils has been studied mainly at relatively large Reynolds numbers, $R e>10^{6}$ (see Refs. 58-60, where the emphasis is on modeling stall and flow reattachment. Dynamic stall studies on NACA 0018 airfoils at $R e<3 \cdot 10^{5}$ are unusual ${ }^{55}$ ).

The first objective of this investigation is to describe a combined theoretical, numerical and experimental study aimed at validating the theories of Greenberg and Isaacs for large amplitude free stream oscillations of $\sigma=0.5$. Three different theoretical approaches to predict the overshoot are compared and the ability of URANS to capture the unsteady flow physics is assessed. For this purpose, the lift, the leading-edge pitching moment, and the pressure distribution along the chord are valuable parameters for comparison. The second objective is to experimentally and computationally study dynamic stall under idealized conditions representative of wind turbine rotor blades. Dynamic stall occurs suddenly and is influenced by the pressure gradient along the chord, the boundary layer, the global dynamics in the system (expressed by the reduced frequency), etc. This is a challenging setup for CFD and the conducted experiments represent a great basis for validation.

\section{Description of CFD code used for unsteady flow predictions}

Alongside the experimental effort and Isaacs' theory, a companion CFD study is being conducted for the same configurations. At this time, two-dimensional steady RANS and unsteady URANS calculations have been employed for several different cases. The latter computations include simulation of the unsteady free stream and moving airfoil, and are compared with the experimental data and theory. Also, a threedimensional steady RANS calculation including the test section (side walls, floor and ceiling) at several angles of attack has been performed in order to explore the three-dimensionality of the flow at higher lift conditions. Due to the higher computational cost of the three-dimensional calculations, this has not yet been extended to unsteady flows.

The structured grid CFD code CFL3D ${ }^{61}$ is employed for the RANS and URANS computations. It is an upwind finite volume code that solves the full Navier-Stokes equations. CFL3D can solve multiple zone structured grids that are connected in an one-to-one, patched, or overset manner. It possesses the SpalartAllmaras (SA), ${ }^{62}$ Menter SST, ${ }^{63}$ and SSG/LRR-RSM-2012 second moment Reynolds stress ${ }^{64}$ turbulence models (among others) to simulate turbulent flows. For all results to be shown in this paper, only the SA model was employed. It was run as fully turbulent, with no presumed transition on the airfoil. CFL3D is globally second order spatially accurate, and can be used to solve time accurate problems with moving bodies and with flow control applied at walls. ${ }^{65}$

For time accurate problems, CFL3D utilizes pseudo time stepping and achieves second order temporal accuracy. With pseudo time stepping, sub-iterations are used to reduce the linearization and factorization 
errors, and advance the solution in pseudo time to the next physical time.

For time accurate URANS computations, the decomposition for forced periodic cases can be written as

$$
F(x, t)=\langle F(x, t)\rangle+F^{\prime}(x, t)
$$

where $F(x, t)$ is the total signal, the $\langle F(x, t)\rangle$ term is the phase average, and $F^{\prime}(x, t)$ is the turbulence. This form resembles standard Reynolds decomposition, except that the flow is split into a slowly varying mean (phase average) and a random fluctuating part. As a result, the final conservation equations in terms of phase averaged variables $\langle F(x, t)\rangle$ are identical in form to the standard Reynolds averaged equations. In other words, when URANS is used for a forced periodic flow field such as that produced by oscillations of the airfoil or free stream perturbations, the computation (depending on the turbulence model and the case) often eventually settles down to a nearly exactly repeatable periodic variation. If and when this repeatability is attained, any single point during the cycle corresponds to a phase averaged result from the experiment.

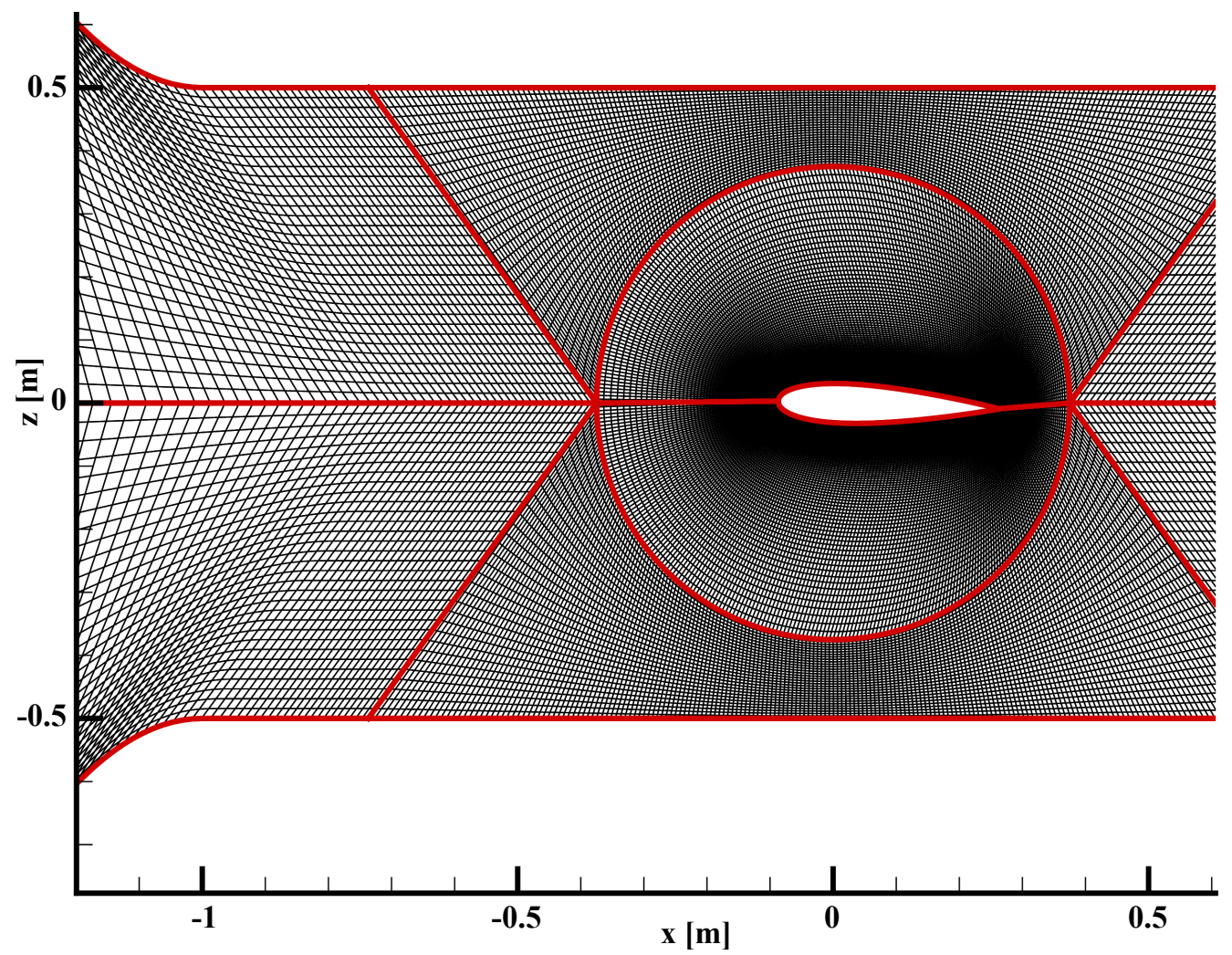

Figure 1. Part of the tunnel grid with the NACA 0018 airfoil used by CFD (zone boundaries are shown in bold red lines).

In order to include the varying free stream speed in the CFD, at the outflow of the tunnel grid the boundary condition sets a sinusoidal time varying back pressure, resulting in variable speed through the tunnel. This strategy attempts to closely mimic the tunnel conditions. However, several difficulties have been noted with this approach. First, it is a trial-and-error process to discover the back pressure mean magnitude $p$ and change $\Delta p$ that will yield a desired tunnel velocity variation. Second, the conditions achieved are a strong function not only of the back pressure, but also of the frequency of oscillation. In other words, at one frequency a certain $p$ and $\Delta p$ will produce the desired tunnel velocity variation, but at a different frequency the same numbers no longer work, and a new set must be found by trial-and-error. And finally, when performing simultaneous tunnel speed variation and airfoil pitching, the phase of the tunnel back pressure must be adjusted so that the tunnel velocity is phased appropriately with the airfoil pitching.

For simulating the airfoil pitching, circular grid zones were constructed around the airfoil, and these were dynamically rotated during the computation. In this process, information is passed to/from the surrounding tunnel grid via patched interfaces. A picture of the fine grid is shown in figure 1, focusing on the region near and just upstream of the airfoil. The two zones with circular outer boundary are rotated to $\alpha=2^{\circ}$ in this 
view. The grid includes the tunnel's upstream settling chamber and contraction; the inflow in the grid is at $x=-4.5 \mathrm{~m}$, and the contraction ratio is 7.91 . The outflow location is at $x=3.77 \mathrm{~m}$. Note in the CFD that $z$ is "up" and $y$ is spanwise.

The boundary conditions at the airfoil surface are viscous (no-slip) adiabatic. The tunnel top and bottom walls are simulated via inviscid slip-wall conditions. At the tunnel outflow, the pressure $\left(p / p_{\text {ref }}\right)$ is specified (either constant or sinusoidally varying, depending on the case), while all other quantities are extrapolated from the interior of the domain. At the tunnel inflow, the total pressure and total temperature are specified at free stream (reference) levels, and the Riemann invariant is extrapolated from the interior of the domain.

\section{Experimental replication of blade conditions}

The wind tunnel is driven by a $75 \mathrm{~kW}$ blower, has an 8:1 contraction ratio and a top wind speed of $55 \mathrm{~m} / \mathrm{s}^{50,51}$ The wind tunnel was designed specifically to accommodate smooth flow speed variations by selecting a backward bladed impeller that operates smoothly even when fully stalled. Flow distortion in the upstream test section and wind tunnel turbulence are less than $0.2 \%$ and $0.1 \%$ respectively. The test section is equipped with large rings that incorporate transparent Plexiglas ${ }^{\circledR}$ windows (see figure 2). The rings are driven by a servomotor via a series of two steel reinforced belt drives. In addition, the floor and ceiling are also made of Plexiglas ${ }^{\circledR}$ rendering full optical access to the test section. In this study, the NACA 0018 airfoil is attached to, and mounted between, the Plexiglas ${ }^{\circledR}$ walls. The rings are able to perform arbitrary pitching motion of the airfoil through a full $360^{\circ}$ at maximum rate of $150^{\circ} / \mathrm{s}$. This custom built test section with arbitrarily large angle of attack range, combined with high pitching speeds and full optical access renders this setup unique. The system was programmed using LabVIEW ${ }^{\mathrm{TM}}$ with the so-called CompactRIO ${ }^{\mathrm{TM}}$, which is a realtime industrial controller made by National Instruments. Considerable expense and manpower has been invested in perfecting and validating this test section. Design of the system took into account the stiffness of the belts and motor response to the loads. At peak loads, the maximum error was calculated to be $<0.25^{\circ}$; direct measurements confirmed the fidelity of the design with errors never exceeding $0.2^{\circ}$.

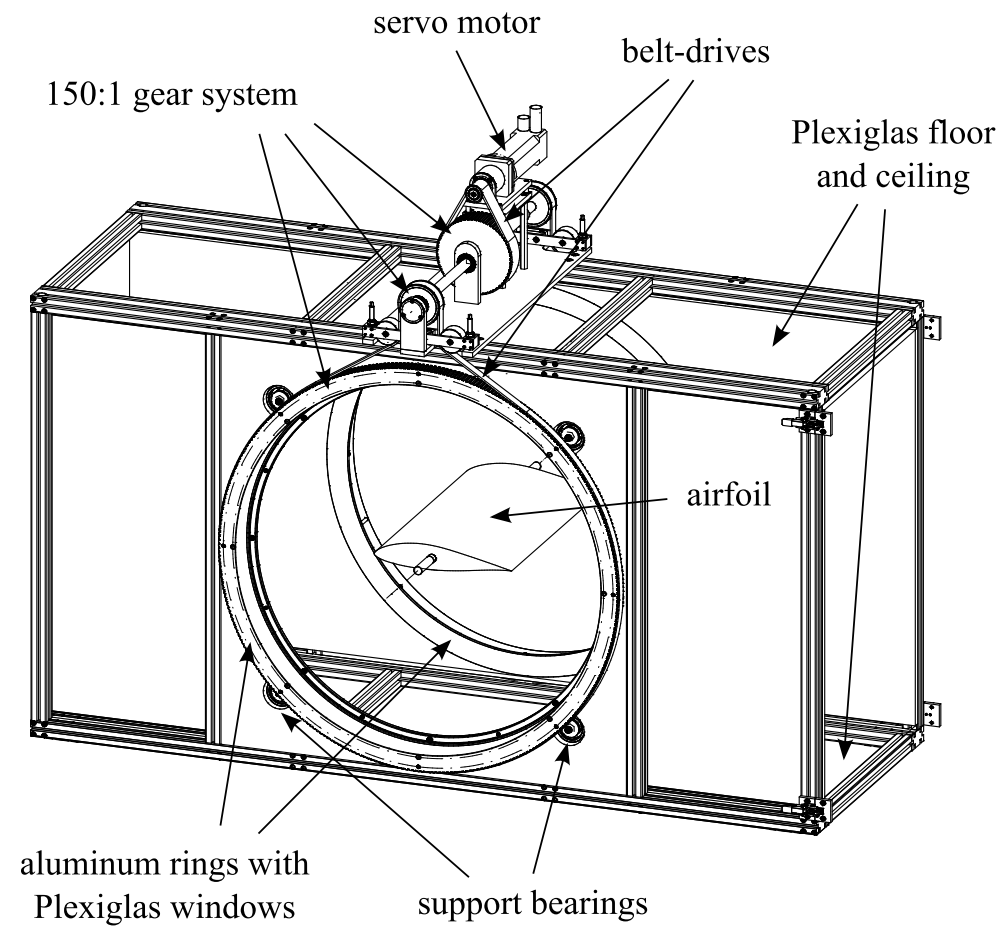

Figure 2. View of the test section showing the servomotor and belt drives as well as the approximate airfoil location.

The well-known method of employing louver vanes was adopted for the present tunnel. The vanes dynamically vary the cross sectional exit area of the tunnel and thus, vary the head-loss resulting in unsteady velocity oscillations. The present implementation in figure 3 involves a louver mechanism with 13 counter 
rotating vanes driven by a $0.75 \mathrm{~kW}$ servo motor with a $5: 1$ gear ratio, coupled with PLC controller. This enables the dynamic control of the vanes' position, and the programming of various arbitrary rotation profiles, thus creating different temporal velocity profiles inside the upstream test section. A comparison of the measured, sinusoidal velocity profile with an ideal sine yield a maximum deviation of $2 \%$ at a velocity ratio of $0.5{ }^{66}$ Thus, the entire free stream velocity profile is expected to be sufficient for dynamic measurements.

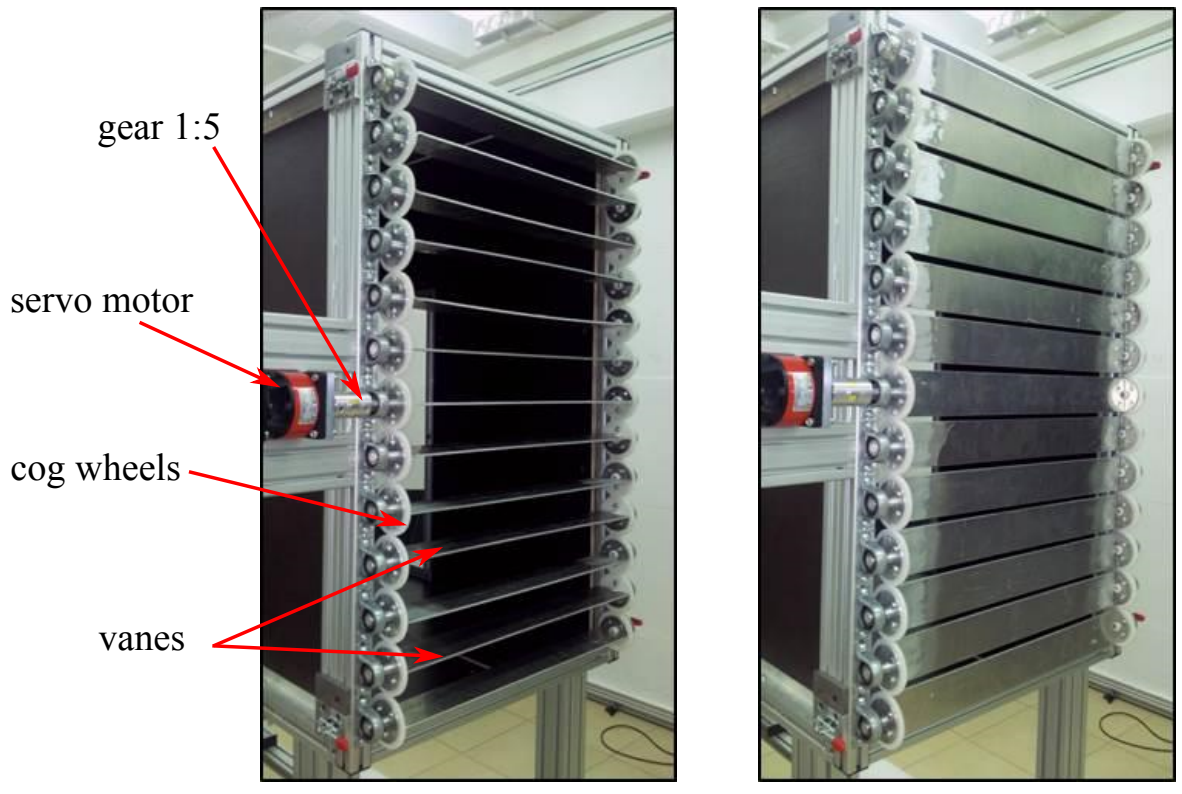

Figure 3. Photograph of the 13 louver vans at the end of the test section in open and closed position.

The lift is calculated by means of 40 pressure taps located on the centerplane of the model. The measured static pressure, which acts normal to the surface, is weighted by the half distance to the neighboring pressure taps and transformed in the coordinate system of the wing chord. The summation yields the lift and the pressure drag (drag due to friction is not quantified). The cross product of the static pressure at each pressure tap and the distance to the leading edge gives the leading edge pitching moment. The phase reconstruction is based on the averaged free stream velocity of the two hotwires. Taking into account that the amplitude of the free stream oscillation varies slightly, each single period was fitted by an ideal sine to avoid any unphysical scatter in the data. Each measurement consists of at least 150 periods. The data are averaged at each $\alpha_{\text {step }}=0.5^{\circ}$ with a window size of $\pm 0.3^{\circ}$ and at each $\phi_{\text {step }}=2^{\circ}$ with a window size of $\pm 1^{\circ}$.

\section{Theoretical unsteady lift prediction in pre-stall configuration}

As discussed above, unsteady conditions may yield significant unsteady lift effects on airfoils even when the flow remains fully attached. For this scenario, several theories predict the lift response assuming a flat plate in incompressible potential flow. ${ }^{2-4,9,10,13,14}$ The assumed inflow and the airfoil geometry are homogenous and two-dimensional. The infinite wake itself is planar, starts at the trailing edge and no diffusion is considered. The wake convects downstream along the $x$-axis without any vertical or horizontal displacement due to self induction. The amplitude of the oscillating free stream is limited to values $\sigma<1$ to avoid reverse flow. The assumption of potential flow requires fully attached flow in experiments for a valid comparison. Thus, trailing edge stall or laminar separation bubbles lead to significant deviations.

For a pure harmonic pitching motion in constant free stream, Theodorsen ${ }^{2}$ developed a closed form solution to predict the unsteady lift overshoot. It includes the so called Theodorsen functions $C(n k)=$ $F(n k)+i G(n k)$, which represent the basis for all further solutions. The unsteady lift generated by a sinusoidal angle of attack oscillation $\alpha(\phi)=\alpha_{0}+\beta_{0} \sin (\phi)$ is given in equation (2). The steady lift is defined as $L_{0}=\pi \rho c u_{s}^{2} \alpha_{0}$.

$$
\frac{L(\phi)}{L_{0}}=2 \pi\left(0.5 k\left(\beta_{0} \cos (\phi)+a k \beta_{0} \sin (\phi)\right)+\alpha_{0}+\beta_{0} \sin (\phi) F(n k)\right.
$$




$$
\left.-k(0.5-a) \beta_{0} \sin (\phi) G(n k)+\beta_{0} \cos (\phi) G(n k)+k(0.5-a) \beta_{0} \cos (\phi) F(n k)\right)
$$

Considering an airfoil at a constant angle of attack exposed to an unsteady free stream, Greenberg ${ }^{10}$ and Isaacs ${ }^{3}$ provided solutions to predict the unsteady lift response. However, for large relative velocity amplitudes of $\sigma>0.4$, Greenberg's approach yields significant deviations from theory ${ }^{4}$ and experiments. ${ }^{66}$ Thus, only Isaacs' results are discussed here briefly and shown in equation (3), assuming a sinusoidal free stream $u(\phi)=u_{s}(1+\sigma \sin (\phi))$. The lift coefficient from this equation is used instead of the lift coefficient from $\frac{C_{l}(t)}{C_{l, q s}}=\frac{L(t)}{L_{s}} \frac{1}{(1+\sigma \sin (\omega t))^{2}}$. This interpretation is independent of the dynamic pressure variations of the free stream and hence represents the pure unsteady lift response.

$$
\begin{aligned}
\frac{C_{l}(t)}{C_{l, q s}}= & \frac{1}{(1+\sigma \sin (\omega t))^{2}}\left[1+0.5 \sigma^{2}+\sigma\left(1+\Im\left(l_{1}\right)+0.5 \sigma^{2}\right) \sin (\omega t)\right. \\
& \left.+\sigma\left(\Re\left(l_{1}\right)+0.5 k\right) \cos (\omega t)+\sigma \sum_{m=2}^{\infty}\left(\Re\left(l_{m}\right) \cos (m \omega t)+\Im\left(l_{m}\right) \sin (m \omega t)\right)\right]
\end{aligned}
$$

Note that $l_{m}$ includes an infinite summation, and $J$ represents Bessel functions of the first kind.

$$
\begin{aligned}
& l_{m}=-m(-i)^{m} \sum_{n=1}^{\infty}\left[F_{n}\left(J_{n+m}(n \sigma)-J_{n-m}(n \sigma)\right)+i G_{n}\left(J_{n+m}(n \sigma)+J_{n-m}(n \sigma)\right)\right] \\
& \left.\begin{array}{l}
F_{n} \\
G_{n}
\end{array}\right\}=\frac{J_{n+1}(n \sigma)-J_{n-1}(n \sigma)}{n^{2}}\left\{\begin{array}{l}
F(n k) \\
G(n k)
\end{array}\right.
\end{aligned}
$$

Isaacs was the first to publish a closed form solution for the combined case of a sinusoidal free stream oscillation and a harmonically pitching wing. ${ }^{9}$ Based on this approach, van der Wall generalized Isaacs' ansatz and included plunge motion and an arbitrary location of the pitch axis. ${ }^{4}$ The unsteady lift response for a sinusoidal free stream $u(\phi)=u_{s}(1+\sigma \sin (\phi))$ and an in-phase pitching motion $\alpha(\phi)=\alpha_{0}\left(\overline{\alpha_{0}}+\alpha_{1 s} \sin (\phi)\right)$ is given in equation (6).

$$
\begin{aligned}
\frac{C_{l}(\phi)}{C_{l, q s}}= & (1+\sigma \sin (\phi))^{-2}\left[\left(\left(1+0.5 \sigma^{2}\right) \overline{\alpha_{0}}+\sigma \alpha_{1 s}\right)(1+\sigma \sin (\phi))+\sum_{m=1}^{\infty}\left(l_{m} \cos (m \phi)+l_{m}^{\prime} \sin (m \phi)\right)\right. \\
& \left.+0.5 k\left(\left(\sigma \overline{\alpha_{0}}+\alpha_{1 s}\right) \cos (\phi)+k a \alpha_{1 s} \sin (\phi)+\sigma \alpha_{1 s} \sin (\phi)\right)\right]
\end{aligned}
$$

Due to the combined occurrence of the two degrees of freedom, the definition of $F_{n}+i G_{n}$ changes and includes more parameters. ${ }^{4}$

$$
\begin{aligned}
F_{n}+i G_{n} & =n^{-2}[F(n k)+i G(n k)]\left(H_{n}+i H_{n}^{\prime}\right) \\
H_{n} & =0.5\left(J_{n+1}-J_{n-1}\right)\left(\sigma \overline{\alpha_{0}}-\alpha_{1 s}\right)-(n \sigma)^{-1}\left(2 J_{n} \alpha_{1 s}\right) \\
H_{n}^{\prime} & =\sigma^{-1} J_{n}\left(-k(0.5-a) \alpha_{1 s}\right)
\end{aligned}
$$

\section{Results}

In a first step, the baseline of the NACA 0018 experiments and the two- and three-dimensional CFD simulations are described. Then, the unsteady lift response in fully attached flow at low angles of attack are presented. An oscillating free stream, pitching motion and the combination of both unsteady effects are compared to theoretical predictions. Finally, implications for the airfoil pitched beyond the static stall angle are discussed.

In all CFD cases, both medium and fine grids were tested, and the differences in results were found to be minimal. Unless otherwise noted, fine grid results are shown for all cases. For unsteady flows, the CFD also explored the effects of number of subiterations (per physical time step) and time step value itself on the results. Generally, use of at least 10 subiterations in combination with a time step yielding at least 600 steps per period were found to be sufficient. Unsteady computations were initiated from steady-state solutions, and continued until a repeatable periodic variation was reached (typically after $2-3$ cycles). 


\section{A. Steady results from CFD and experiment}

In order to assess the angle of attack range over which CFD is likely to be reliable, steady CFD results were obtained over a range of angles of attack and compared with experimental data. Results are shown in figure 4. Two different grid sizes were employed to assess the influence of discretization errors. The fine grid (f) had 97,280 grid cells, and the medium grid (m) had 24,320 grid cells. As seen in figure 4, there was little influence of grid size on the resulting lift coefficient.

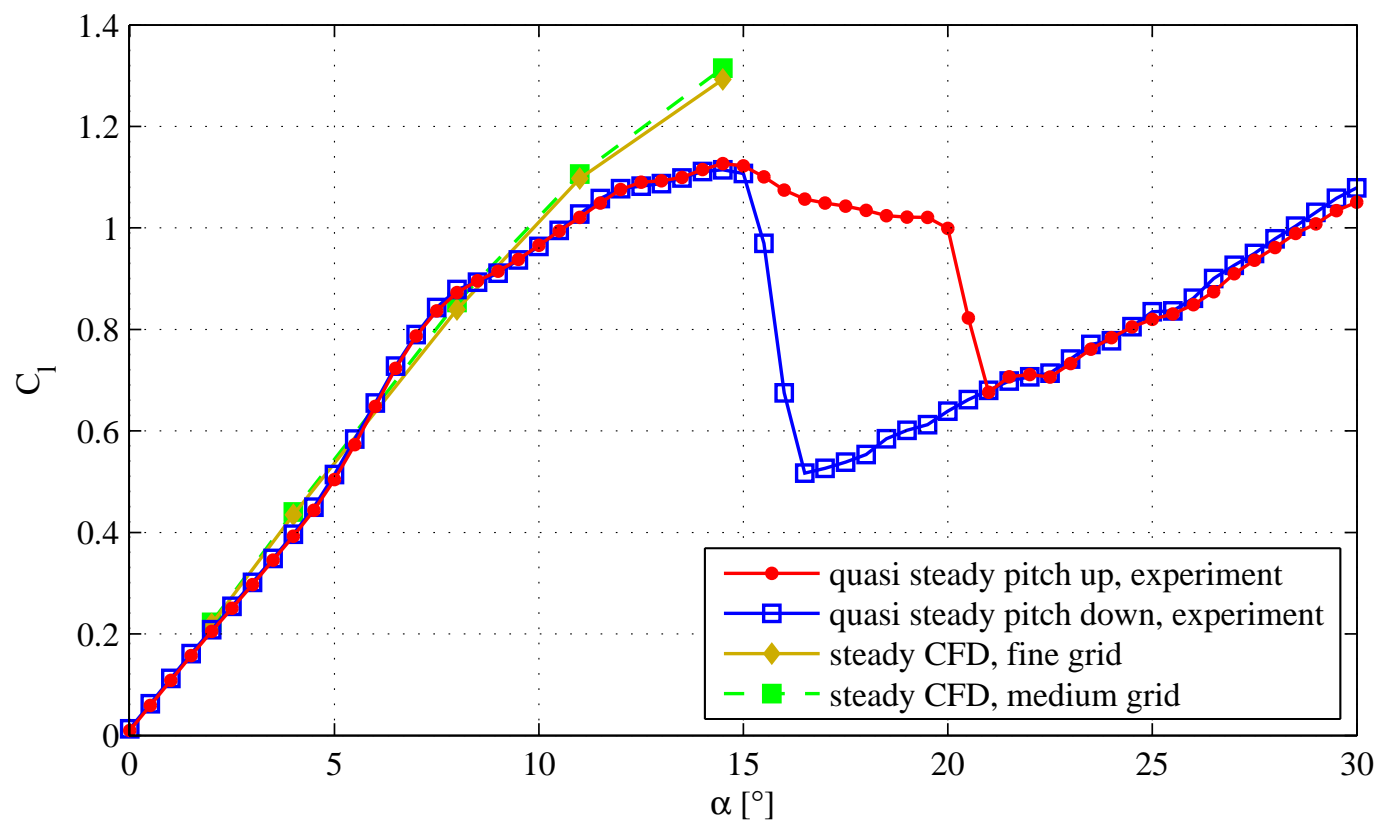

Figure 4. Steady lift coefficients of a NACA 0018 elaborated by quasi-steady experiments (continuous pitch sweeps) and two-dimensional steady CFD at $R e=3 \cdot 10^{5}$.

In the experiment, angles of attack well beyond stall were considered, and hysteresis was noted in the range $15^{\circ} \leq \alpha \leq 21^{\circ}$, as seen in figure 4 . Data were acquired using continuous pitch sweeps at very low $k=0.000085$. The maximum lift coefficient $C_{L}$ (near $\alpha=14.5^{\circ}$ ) was around 1.1. In CFD, results started to deviate from the experiment above $\alpha$ of $8-10^{\circ}$. Near here, the lift coefficient slope of the experiments was reduced significantly below the theoretical slope (assuming potential flow) of $2 \pi$, suggesting the onset of trailing edge stall. At $\alpha=14.5^{\circ}$, the computed $C_{L}$ of about 1.3 significantly overpredicted the experimental value. There is no doubt that two-dimensional CFD is incapable of matching the experiment at higher angles of attack, so the two-dimensional steady computations were taken no further than $14.5^{\circ}$.

Two three-dimensional steady RANS computation were run at $\alpha=2^{\circ}$ and $14.5^{\circ}$, with viscous tunnel side walls included. For these computations, the two-dimensional fine grid was extended in the spanwise direction using 64 grid cells over $y=0.305 \mathrm{~m}$ (half the full tunnel width), for a total grid size of 6.2 million grid cells. The grid was clustered at the side wall, where viscous adiabatic boundary conditions were imposed. At the centerplane, symmetry boundary conditions were imposed.

Surface pressure coefficient contours along with surface streamline patterns are shown in figures 5 and 6 . At $\alpha=2^{\circ}$, the flow is attached and two-dimensional over most of the wing, with only a very small region of three-dimensionality very near the side wall. At the centerplane, the flow was fully attached, satisfying the Kutta condition. This enables a comparison to the previously discussed theories that assume a flat plate in potential flow. Hence, this setup at low angles of attack provides a unique possibility of validating CFD, experiments and theory. Integration of the CFD surface pressure coefficients along the centerplane of the 3 -D computation yield a lift coefficient value per unit span of approximately 0.203 , which is very close to experiment and to the 2-D computational result shown in figure 4.

At the higher angle of attack of $\alpha=14.5^{\circ}$, there is clearly a substantial influence of the side wall, as seen in figure 6 . Note that this computation predicts significant regions of separated flow, and is not fully converged; therefore, this result should be viewed only qualitatively. Even though the flow appears to be 


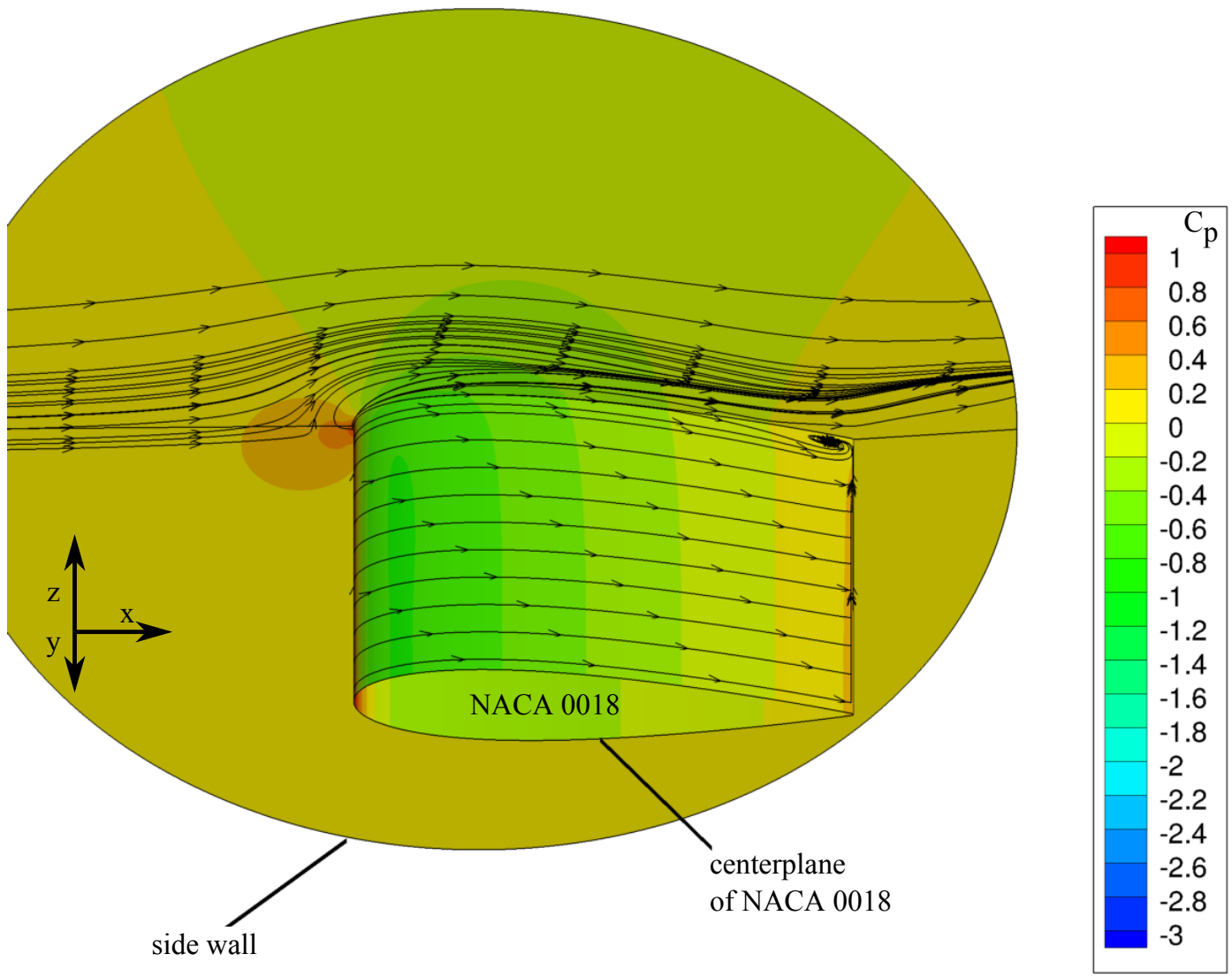

Figure 5. Surface pressure coefficient contours and surface streamlines for three-dimensional steady computation on fine grid at $\alpha=2^{\circ}$.

reasonably two-dimensional over a large portion of the central region of the wing, the three-dimensionality has still affected the flow in that region. This can best be seen in a plot of surface pressure coefficient taken at the center plane position. In figure 7 , the three-dimensional computed $C_{p}$ is compared to results from the two-dimensional computation. The three-dimensional results show significantly lower suction values, in better agreement with experiment at the center plane location. Integration of the CFD surface pressure coefficients along the centerplane of the 3-D computation yield a lift coefficient value per unit span of approximately 1.17. This value is much closer to experiment than the 2-D computational result.

A similar influence of three-dimensional sidewall structures on the assumed two-dimensional flow near the tunnel centerplane was noticed previously in Rumsey et al. ${ }^{67}$ for a high lift multi-element airfoil. In other words, a local region of apparent two-dimensionality over a region of a wing in a tunnel is not a guarantee of two-dimensional flow in the purest sense of the word. Three-dimensional structures near the side walls still influence the entire flow field (typically the higher the angle of attack, the greater the influence).

The experimental data of the quasi steady pitch up and pitch down do not show any appreciable deviations at $\alpha=14.5^{\circ}$ (figure 7); i.e., no hysteresis effects exist at this angle of attack. Furthermore, the measured pressure distribution suggests the possible presence of a laminar separation bubble at approximately $x / c=$ 0.07. Alternatively, the slight deviation in the surface pressure distribution may be attributed to a spanwise blowing slot (height $1.2 \mathrm{~mm}$ ) that can be used for active flow control. Even though the entire slot was sealed with thin adhesive tape, ${ }^{51}$ the surface discontinuity may have altered the transition process. Furthermore, figures 6 and 7 both suggest trailing edge separation over a broad region of the airfoil. At the trailing edge itself, reverse flow occurs and the Kutta condition is violated. This renders any comparison to potential flow theory impossible at these high angles of attack. 


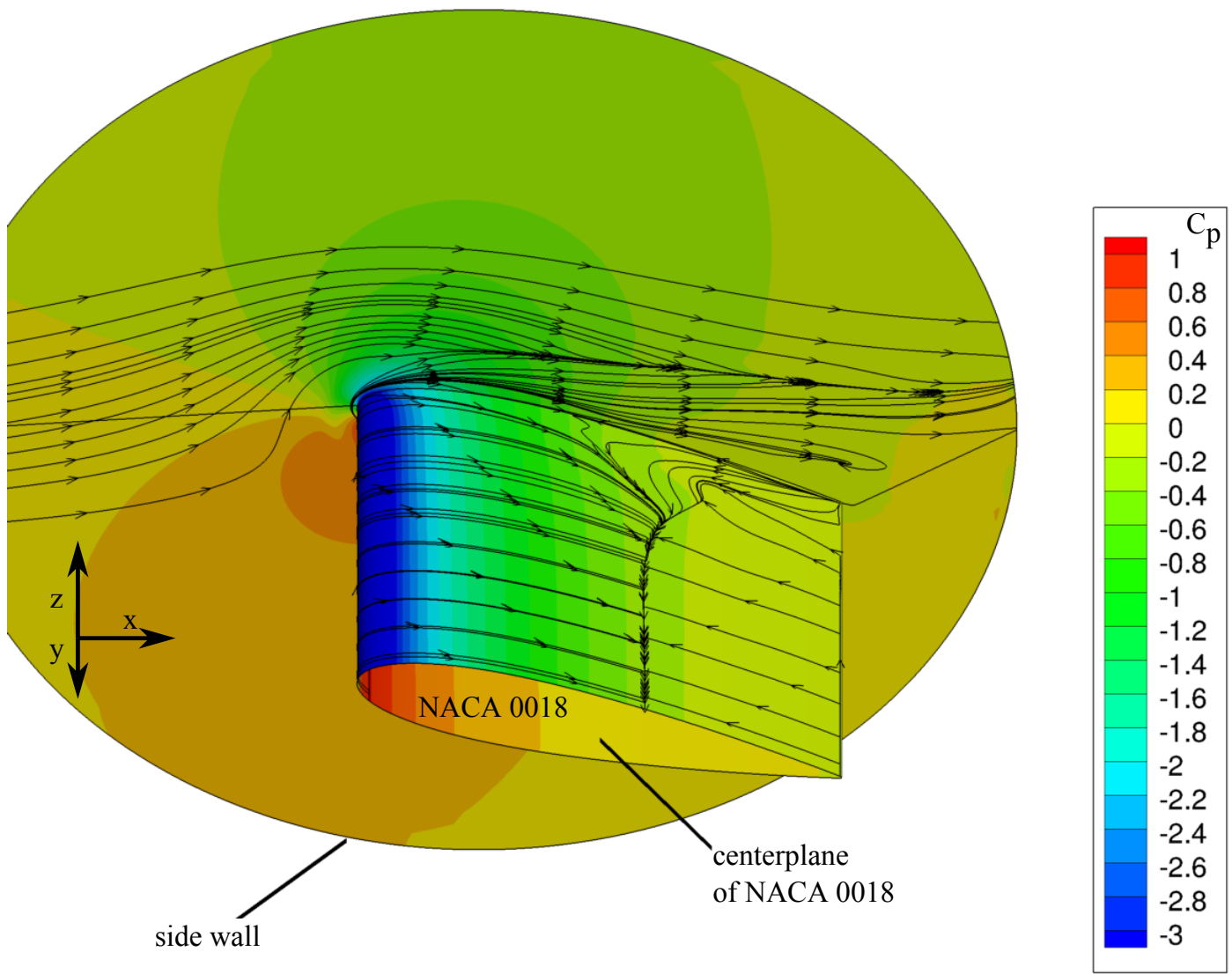

Figure 6. Surface pressure coefficient contours and surface streamlines for three-dimensional steady computation on fine grid at $\alpha=14.5^{\circ}$.

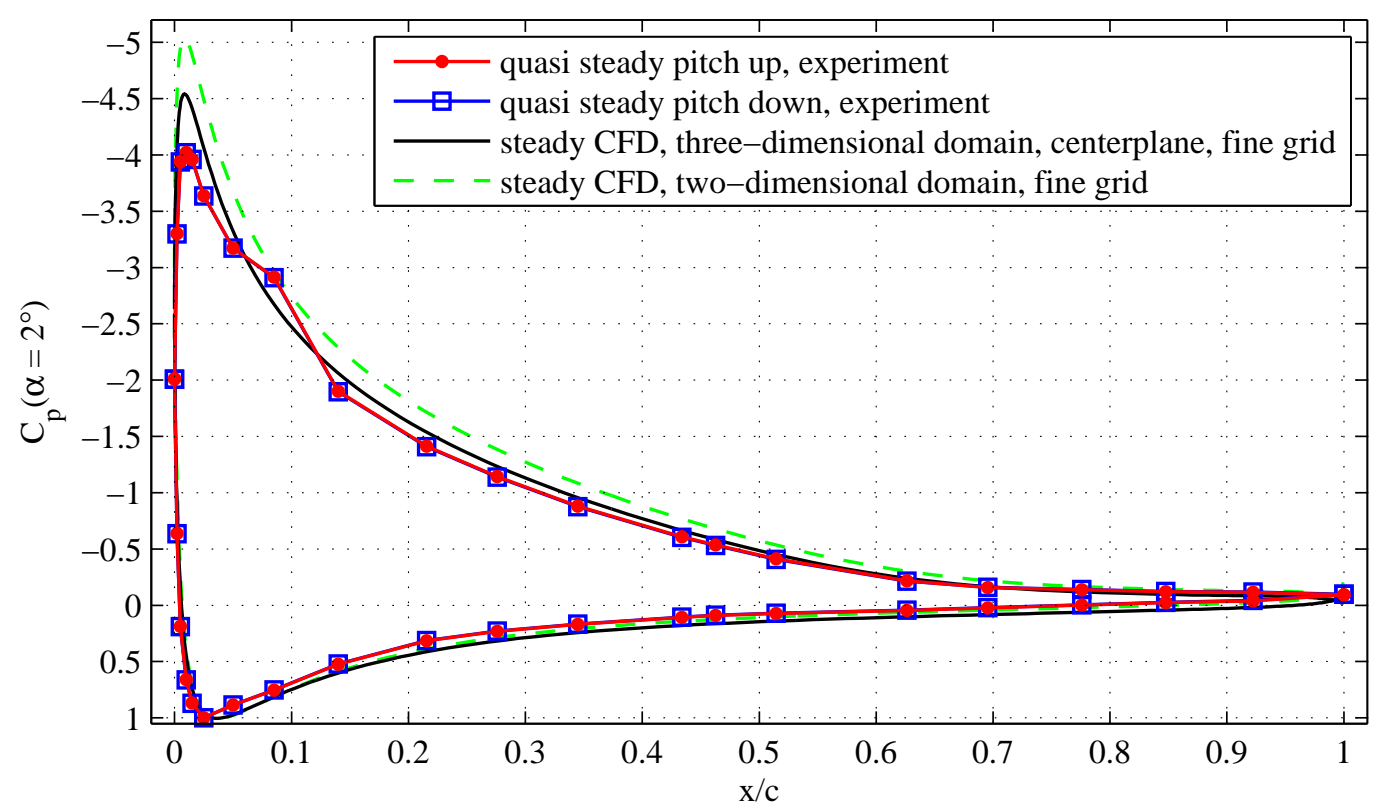

Figure 7. Steady surface pressure coefficients, NACA 0018, $\alpha=14.5^{\circ}$. 


\section{B. Comparison of the unsteady lift overshoot of a NACA 0018 facing an oscillating free stream}

Validation of both the experimental and computational approaches in relatively simple fully attached flows was considered important. The first step was an investigation of the influence of an unsteady freestream on an airfoil at a fixed low angle of attack. A comparison to experiments and potential flow theory assesses the reliability of 2-D URANS.

The results are presented in the form of lift coefficient ratio, as shown in figure 8 . This kind of presentation compensates for the strong dynamic pressure variations during the free stream velocity variations; and hence, only the net unsteady effects are highlighted. Quasi steady experiments were performed by slowly varying the wind tunnel speed at $k \approx \mathcal{O}\left(10^{-4}\right)$. In the $\mathrm{CFD}$, reproducing quasi steady conditions was computationally difficult because the boundary conditions needed to be determined iteratively at each time step, as described earlier. Hence, steady computations were performed instead at 22 corresponding free stream conditions, and then a 6th-order best-fit polynomial was used to create an estimate for the quasi steady CFD lift variation. The unsteady CFD runs, yielding $C_{l}$ as a function of $\phi$, were conducted at the appropriate $k$ by time-varying the tunnel back pressure in order to achieve the desired tunnel velocity variation.

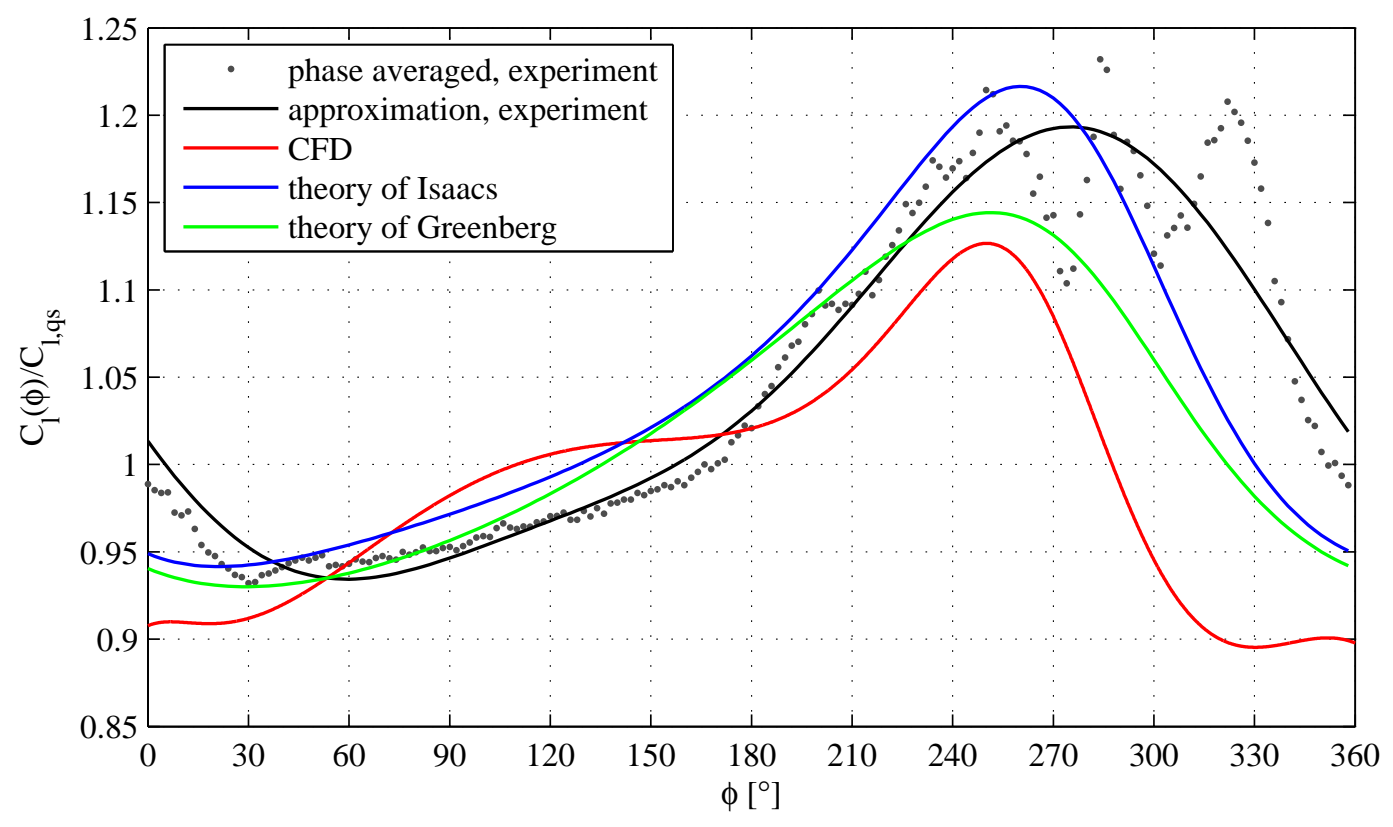

Figure 8. Experiments, CFD, and theory for the unsteady to steady lift ratio as a function of phase angle $\phi$ for a NACA 0018 at $\overline{R e}=2.5 \cdot 10^{5}$ and $\alpha=2^{\circ}$ subjected to an unsteady free stream oscillation of $\sigma=0.5$ and $k=0.074$.

In this particular case, the NACA 0018 is exposed to a sinusoidal free stream oscillation with an amplitude of $50 \%$, resulting in $u(\phi)=u_{s}(1+0.5 \sin (\phi))$. The time averaged free velocity is $u_{s}=11.1 \mathrm{~m} / \mathrm{s}$ which corresponds to $\overline{R e}=2.5 \cdot 10^{5}$. The angle of attack was kept constant at $\alpha=2^{\circ}$ and the reduced frequency was $k=0.074$. Over the first half of the cycle (when freestream velocity is high), the lift experiences a deficit compared to quasi steady values. Over the second half of the cycle (when freestream velocity is low), the trend reverses and the lift exhibits overshoots compared to quasi steady values.

In figure 8 , near the maximum lift overshoot, the experiments (shown as black dots) exhibited an oscillation at $260^{\circ} \leq \phi \leq 320^{\circ}$. This phenomenon possessed a high frequency and the corresponding reduced frequency is approximately $k=0.7$. By means of measured, phase averaged pressure distributions in this range, this was traced to the formation and shedding of a recirculation bubble directly upstream of the trailing edge. This high frequency phenomena affected the flow field at the trailing edge by modifying the Kutta condition. Thus, the circulation varies and the shed wake vortex sheet is modified as well to keep the global circulation constant in the system. Finally, the modified wake vortex distribution leads to another measured lift overshoot compared to the theory. It is not clear whether this is caused by low Reynolds number effects, the relatively thick airfoil, or their combination. The solid black line represents an approximation of the 
experimental results by a Fourier series including only the first two harmonics.

Regarding the CFD, although the trends are correct, the overall, or integrated, lift enhancing effect is underpredicted. To explain this, the results of the potential flow theories ${ }^{9,10}$ are also presented here. The overall lift enhancement is greater for the theory of Isaacs (blue line), given by equation (3), yielding a better agreement with the experiments. Nevertheless, both theories account for the unsteady shed vorticity at the trailing edge in the form of a semi-infinite wake. Greenberg made a high frequency simplification that is physically equivalent to assuming a quasi steady convection velocity of the shed wake. ${ }^{14}$ Thus, for the relatively high amplitude oscillations of $\sigma=0.5$ considered here, Greenberg's theory is considered less physically representative than Isaacs'. In any case, the modeling of the wake clearly plays a crucial role in predicting the unsteady lift. URANS, however, does not resolve the unsteady flow vorticity, or structures resulting from rollup of the vortices in the wake, because the high values of eddy viscosity tend to "smear" all wake structures.

For example, figure 9 is a snapshot of the unsteady CFD solution. It shows the relatively high levels of eddy viscosity (nondimensionalized by laminar viscosity) in the wake. Mach number contours and vorticity magnitude contours (nondimensionalized by speed of sound and unit length) do not indicate any small-scale wake structures, wake unsteadiness, or significant wake vorticity. Thus, it is hypothesized here that the lower overall lift enhancement predicted by URANS is a direct result of its inability to resolve the wake vorticity. Scale-resolving CFD, such as three-dimensional hybrid RANS/LES methods, in combination with significantly finer grid resolution in the wake, would be required to improve the results.

\section{Comparison of the unsteady lift of a NACA 0018 in oscillating pitching motion}

The second test case considered here is an airfoil pitching at small angles of attack at a constant free stream. The sinusoidal angle of attack profile is $\alpha(\phi)=2^{\circ}+2^{\circ} \sin (\phi)$ at low $k=0.08$ and medium $k=0.263$, with $R e=3 \cdot 10^{5}$. The corresponding theoretical unsteady lift is given in equation (2). Figure 10 shows a comparison of a URANS simulation, experiments and the theory of Theodorsen. ${ }^{2}$ In the case of low $k=0.08$, the slope of the CFD curve is still close to $2 \pi$ and thus, close to the quasi steady baseline. This behavior suggests that the quasi steady variations are too dominant compared to the unsteady lift effects. The theory predicts a reduction of the lift curve slope, which is confirmed by the experimental data. The hysteresis loop starts at $\alpha=2^{\circ}$ in positive direction towards $\alpha=4^{\circ}$. The loops end at approximately $\alpha=1.9^{\circ}$, visualized by the small gap. Although the hysteresis amplitude is similar in all three approaches, the CFD deviates significantly. For example, the CFD reveals a clockwise rotational sense. On the contrary, the experiments and the theory predict a counter-clockwise rotational sense, which is in agreement with the literature. ${ }^{4,68}$ For the higher $k=0.263$, all three approaches yield the same clockwise rotational sense. In theory, the switch occurs at approximately $k=0.16 .{ }^{68}$ All three curves are tilted more horizontally compared to the low $k$ case. Nevertheless, the CFD still shows the highest slope, which is still close to $2 \pi$, suggesting that the CFD behavior is still dominated by the quasi steady changes. On the other hand, the trend of increased hysteresis with increasing $k$ is reproduced by the CFD. Once again, the deficiencies in the URANS may be due to its smearing of the wake vorticity and hence not resolving the unsteady wake structures.

\section{Combined free stream oscillation and pitching motion of a NACA 0018}

In the two previous sections, a pure free stream oscillation and a pure pitching motion were investigated. Now, pitching motion and free stream oscillations occur in-phase, simultaneously and synchronized. Here, both experimental and computational approaches possess some uncertainties, thus, the theoretical unsteady lift is considered as well, using van der Wall's equation (6) based on potential flow theory. This equation delineates a non-linear interaction of the two separated effects. In fact, a simple superposition does not capture the flow physics correctly. To show this, the NACA 0018 is exposed to a sinusoidal free stream $u(\phi)=u_{s}(1+0.5 \sin (\phi))$ and an in-phase pitching motion $\alpha(\phi)=2^{\circ}+2^{\circ} \sin (\phi)$, both individually and collectively. The time averaged free stream velocity is $u_{s}=13.3 \mathrm{~m} / \mathrm{s}$ which corresponds to $\overline{R e}=3 \cdot 10^{5}$. The reduced frequency is $k=0.099$.

Results of the unsteady lift per unit span are shown in figure 11, which compares experiment, theory, and CFD. This plot shows the pure free stream oscillation results at fixed $\alpha=2^{\circ}$, the pure pitching motion results at $0^{\circ} \leq \alpha \leq 4^{\circ}$, and the combination. During the pure free stream oscillation (green lines and symbols), the CFD underestimates the lift between about $0^{\circ}<\phi<130^{\circ}$ and again beyond $\phi \geq 300^{\circ}$. For $130^{\circ}<\phi<300^{\circ}$, the CFD results are close to the experiment and the theory. Regarding the pure pitching 

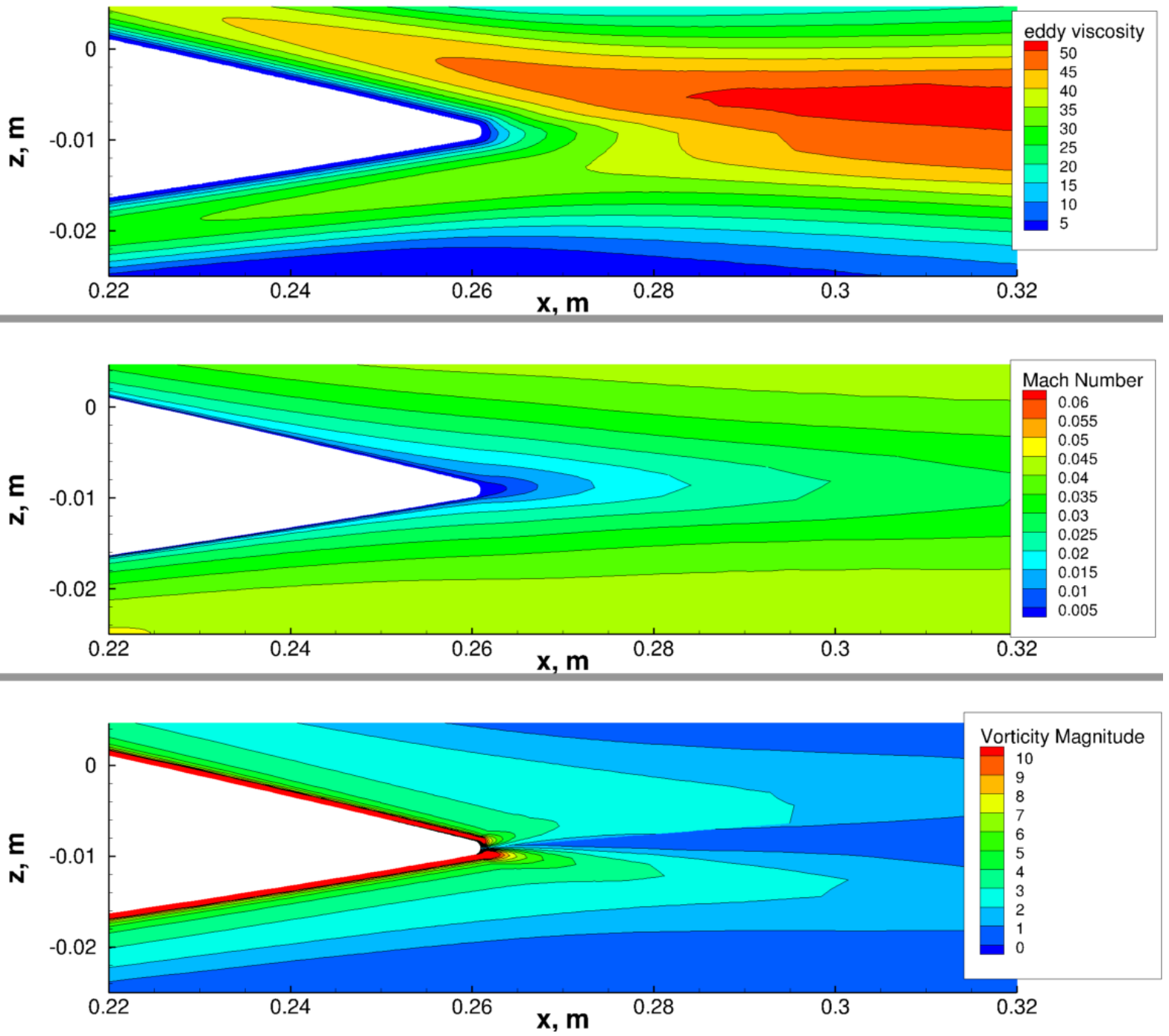

Figure 9. CFD eddy viscosity, Mach number, and vorticity magnitude contours in the wake of a NACA 0018 airfoil at $\alpha=2^{\circ}$ subjected to an unsteady free stream oscillation of $\sigma=0.5$ and $k=0.074$, shown here near $\phi=100^{\circ}$. 

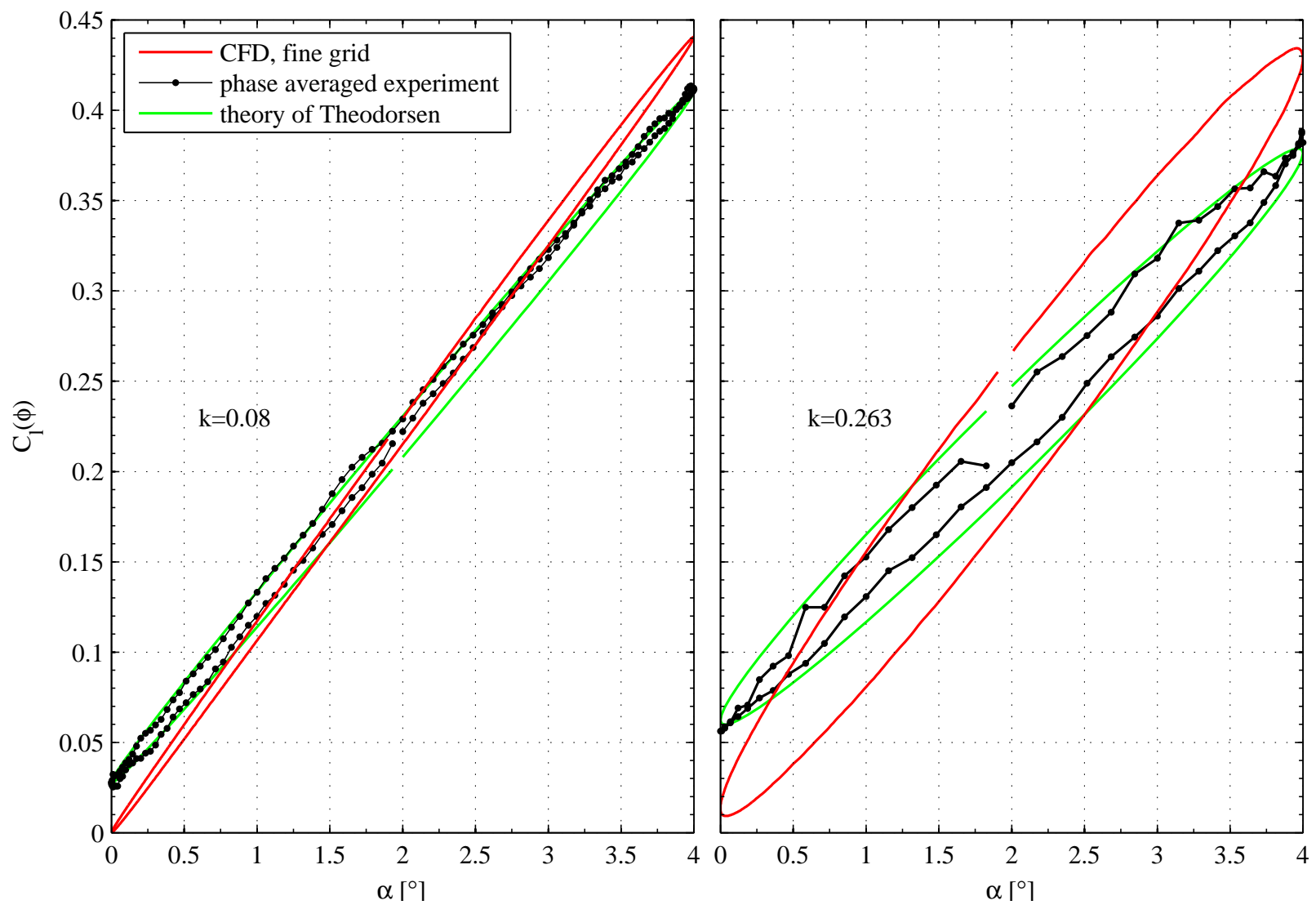

Figure 10. Experiments, CFD, and theory for a NACA 0018 oscillating between $\alpha(\phi)=2^{\circ}+2^{\circ} \sin (\phi)$ at $\operatorname{Re}=3 \cdot 10^{5}$; $k=0.08$ (left) and $k=0.263$ (right). 
motion (blue lines and symbols), the experimental results are almost identical to the theoretical prediction of Theodorsen. ${ }^{2}$ However, similar to the pitching results shown earlier for different $k$, CFD overpredicts the unsteady effects near maximum lift and underpredicts them near minimum lift (the CFD minimum is close to zero).

Considering the synchronized, combined case (red lines and symbols), the overall shape appears to be reasonably well captured by CFD, but again the lift is too close to zero during the second half. Near minimum lift, the CFD is closer to the quasi steady theory using simple superposition (gray line). Near maximum lift, the CFD is lower than experiment while the van der Wall theory is higher than experiment by similar amounts. Both CFD and theory predict significantly lower maximum lift levels than the quasi steady simple superposition, in reasonable agreement with experiment. Thus, it appears that the 2-D URANSalthough able to qualitatively predict the combined effects of surging and pitching - is unable to capture the unsteadiness as accurately as the van der Wall theory, particularly in the range of the lowest angles of attack. A lift of almost zero at $\phi=270^{\circ}$ suggests that the CFD simulation is dominated by the quasi steady effects and the induced lift due to the shed wake vortex sheet has only minor influence. On the other hand, the theory of van der Wall agrees very well with experiment throughout the lift cycle of the combined case.

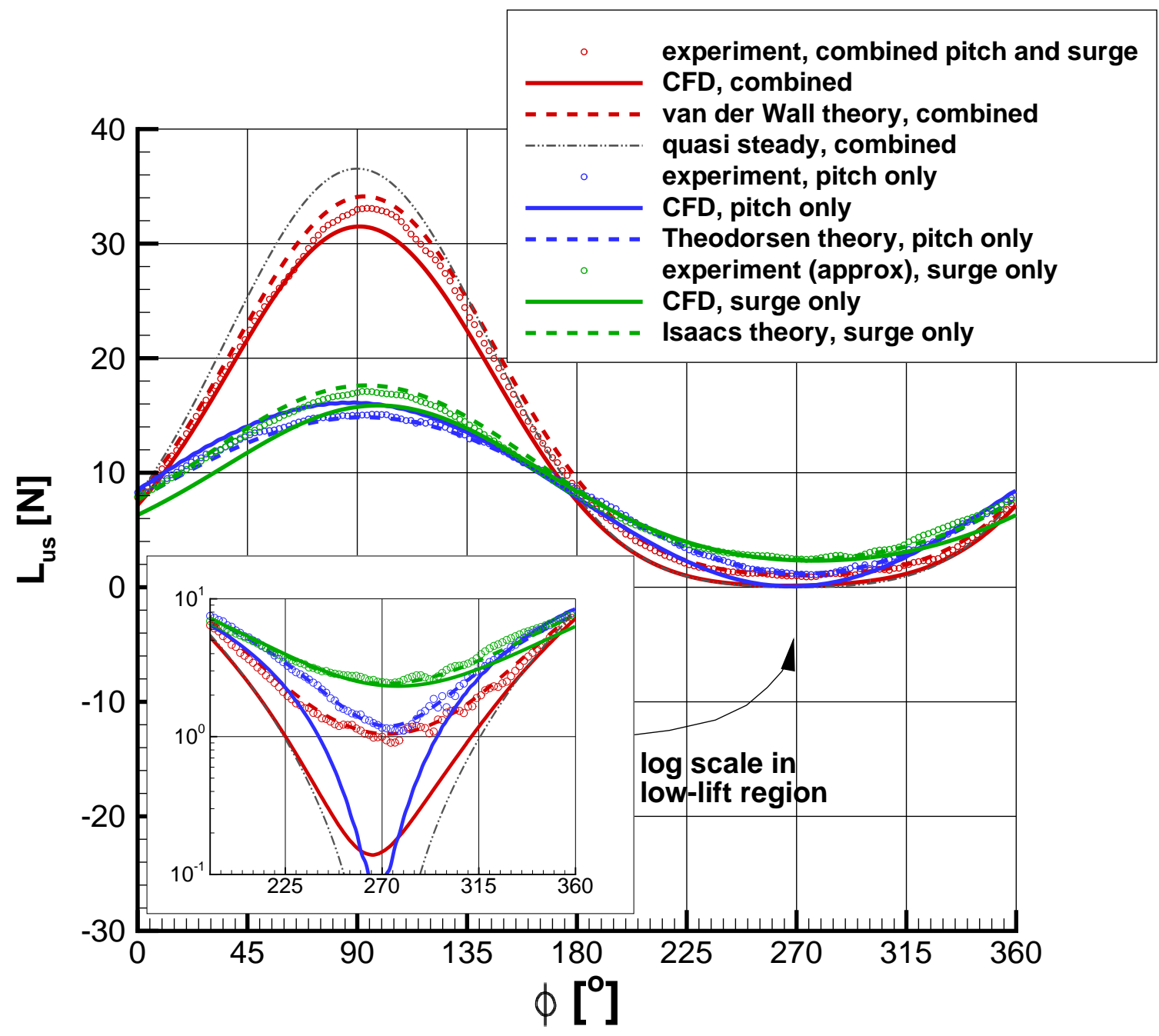

Figure 11. Unsteady lift of a NACA 0018 due to individual and combined/synchronized pitching motion $\alpha(\phi)=2^{\circ}+2^{\circ} \sin (\phi)$ and oscillating free stream $u(\phi)=u_{s}(1+0.5 \sin (\phi))$, with $\overline{R e}=3 \cdot 10^{5}$ and $k=0.099$. 


\section{E. Dynamic stall of NACA 0018}

In preliminary 2-D CFD studies, attempts were made on unsteady dynamic stall pitching cases using $\alpha(\phi)=$ $14.5^{\circ}+3^{\circ} \sin (\phi)$ at Reynolds number $R e=3 \cdot 10^{5}$. As expected, because even the steady calculations at this high of an angle were so far off from experiment (see figure 4), the unsteady pitching results did not have any chance of success. Although not shown here, CFD did show hysteresis, but at much higher lift levels than experiment, close to the quasi steady levels.

Given the earlier steady CFD results, we know that two-dimensional RANS is unable to successfully compute the flows at high angles of attack in the dynamic stall regime. No doubt three-dimensional computations will be required in order to account for the side wall influence. Additionally, RANS/URANS turbulence models themselves are generally known to be poor for predicting flows with large separated regions, and they also smear unsteady wake structures that appear to be important in this context. The shed wake vortex sheet and its upstream induced velocities is the main driver of the unsteady lift response. Considering the three-dimensional CFD results above, the flow on the NACA 0018 wing at $\alpha=14.5^{\circ}$ is separated over as much as $40 \%$ of the upper surface. In this situation, even a three-dimensional URANS computation would likely be unsuccessful. Instead, a three-dimensional hybrid RANS-LES computation would be required, for example. This type of model, which resolves the larger turbulent scales of motion, is known to better capture the physics of massively separated flows, including wake dynamics. However, hybrid RANS-LES can be considerably more time consuming than URANS, so it has not been undertaken to date for this project.

\section{Conclusion}

This three-way experimental, computational and theoretical investigation illustrated a number of important aspects of a thick airfoil subjected to pitching and surging. Pre-stall experiments were conducted at large surge amplitudes, typically encountered on rotorcraft blades; and this proved to be an excellent basis for the validation of Isaacs' theory. The comparison was satisfactory, but near the maximum lift overshoot, the experiments exhibited a high frequency oscillation. This was traced to the formation and shedding of a recirculation bubble upstream of the trailing edge that affected the lift by modifying the Kutta condition. It was not clear whether this is caused by low Reynolds number effects, the relatively thick airfoil, or their combination; future experiments will be aimed at rectifying these two factors. Two-dimensional computations underpredicted the overall lift enhancing effect due to surging because the URANS equations tend to "smear" the wake; on the contrary, the theory explicitly models the wake vorticity.

Excellent correspondence was found between experiments and theory for airfoil pitching as well as combined pitching and surging. In fact, the comparison presented here appears to be the first clear validation of van der Wall's theoretical results. As in the pure surging case, the URANS produced inferior results, presumably again because of its inability to resolve unsteady vortical structures in the wake. Nevertheless, the computations illustrated the importance of conducting full three-dimensional computations when comparing to wind tunnel experiments at high angles of attack, where in particular the tunnel side wall influence needs to be included.

\section{Acknowledgments}

This research was supported in part by the U.S.-Israel Binational Science Foundation (BSF) Grant No. 2012103, by the ISRAEL SCIENCE FOUNDATION (grant No. 840/11) and by the "Stiftung der deutschen Wirtschaft".

\section{References}

\footnotetext{
${ }^{1}$ Barlas, T. and Van Kuik, G., "Review of state of the art in smart rotor control research for wind turbines," Progress in Aerospace Sciences, Vol. 46, No. 1, 2010, pp. 1-27.

${ }^{2}$ Theodorsen, T., "General theory of aerodynamic instability and the mechanism of flutter," NACA rep., No. 496, 1935, pp. 413-433.

${ }^{3}$ Isaacs, R., "Airfoil theory for flows of variable velocity," Journal of the Aeronautical Sciences, Vol. 12, No. 1, 1945, pp. 113-117.

${ }^{4}$ van der Wall, B. G., "The influence of variable flow velocity on unsteady airfoil behavior," Tech. rep., DLR-FB 92-22, Braunschweig, Germany, 1992.
} 
${ }^{5}$ Kutta, W., "Auftriebskräfte in strömenden Flüssigkeiten," Illustrierte Aeronautische Mitteilungen, Vol. 6, 1902, pp. 133135.

${ }^{6}$ Joukowski, N., "On adjoint vortices," Izviestiia, Vol. 13, 1906, pp. 12-25.

${ }^{7}$ Wagner, H., "Über die Entstehung des dynamischen Auftriebes von Tragflügeln," Zeitschrift für Angewandte Mathematik und Mechanik, Vol. 5, No. 1, 1925, pp. 17-35.

${ }^{8}$ Glauert, H., "The force and moment on an oscillating aerofoil," Vorträge aus dem Gebiete der Aerodynamik und verwandter Gebiete, edited by A. Gilles, L. Hopf, and T. Kármán, Springer-Verlag, Berlin, Heidelberg, 1930, pp. 88-95.

${ }^{9}$ Isaacs, R., "Airfoil theory for rotary wing aircraft," Journal of the Aeronautical Sciences, Vol. 13, No. 4, 1946, pp. 218220.

${ }^{10}$ Greenberg, J. M., "Airfoil in sinusoidal motion in a pulsating stream," Tech. rep., NACA TN1326, 1947.

${ }^{11}$ Kottapalli, S. B. R., "Unsteady aerodynamics of oscillating airfoils with inplane motions," American Helicopter Society, Vol. 30, 1985, pp. 62-63.

12 van der Wall, B. G., The influence of variable flow velocity on unsteady airfoil behavior, Master's thesis, University of Maryland, College Park, USA, 1991, UM-AERO 91-46.

${ }^{13}$ van der Wall, B. G. and Leishman, J. G., "The influence of variable flow velocity on unsteady airfoil behavior," $18 t h$ European Rotorcraft Forum, Avignon, France, 1992.

${ }^{14}$ van der Wall, B. G. and Leishman, J. G., "On the influence of time-varying flow velocity on unsteady aerodynamics," Journal of the American Helicopter Society, Vol. 39, No. 4, 1994, pp. 25-36.

${ }^{15}$ Gaunaa, M., "Unsteady 2D potential-flow forces on a thin variable geometry airfoil undergoing arbitrary motion," Tech. rep., Technical University of Denmark, 2006.

${ }^{16}$ Carr, L., "Progress in analysis and prediction of dynamic stall," Journal of Aircraft, Vol. 25, 1988, pp. 6-17.

${ }^{17}$ Ekaterinaris, J. A. and Platzer, M. F., "Computational prediction of airfoil dynamic stall," Progress in Aerospace Sciences, Vol. 33, No. 11, 1998, pp. 759-846.

${ }^{18}$ Florea, R. and Wake, B. E., "Parametric analysis of directed-synthetic jets for improved dynamic-stall performance," AIAA Paper 2003-216, 2003.

${ }^{19}$ Ekaterinaris, J. A., "Numerical investigations of dynamic stall active control for incompressible and compressible flows," Journal of Aircraft, Vol. 39, No. 1, 2002, pp. 71-78.

${ }^{20}$ Joo, W., Lee, B.-S., Yee, K., and Lee, D.-h., "Combining passive control method for dynamic stall control," Journal of Aircraft, Vol. 43, No. 4, 2006, pp. 1120-1128. 2010 .

${ }^{21}$ Marongiu, C. and Tognaccini, R., "Simulation of the dynamic stall at low Reynolds number," AIAA Paper 2010-513,

${ }^{22}$ Martinat, G., Braza, M., Hoarau, Y., and Harran, G., "Turbulence modelling of the flow past a pitching NACA0012 airfoil at $10^{5}$ and $10^{6}$ Reynolds numbers," Journal of Fluids and Structures, Vol. 24, No. 8, 2008, pp. 1294-1303.

${ }^{23}$ Spentzos, A., Barakos, G. N., Badcock, K. J., Richards, B. E., Wernert, P., Schreck, S., and Raffel, M., "Investigation of three-dimensional dynamic stall using computational fluid dynamics," AIAA Journal, Vol. 43, No. 5, 2005, pp. $1023-1033$.

${ }^{24}$ Ferreira, C. S., Kuik, G. V., Bussel, G. V., and Scarano, F., "Visualization by PIV of dynamic stall on a vertical axis wind turbine," Experiments in Fluids, Vol. 46, No. 1, 2009, pp. 97-108.

${ }^{25}$ Ferreira, C., Dixon, K., Hofemann, C., van Kuik, G., and van Bussel, G., "The VAWT in skew: stereo-PIV and vortex modeling," AIAA Paper 2009-1219, 2009.

${ }^{26}$ Tang, D. M., Cizmas, P. G. A., and Dowell, E. H., "Experiments and analysis for a gust generator in a wind tunnel," Journal of Aircraft, Vol. 33, No. 1, 1996, pp. 139-148.

${ }^{27}$ Williams, D., Collins, J., Jankhot, C., Colonius, T., and Tadmor, G., "Control of flow structure on a semi-circular planform wing," AIAA Paper 2008-597, 2008.

${ }^{28}$ Williams, D., Quach, V., Kerstens, W., Buntain, S., Tadmor, G., Rowley, C., and Colonius, T., "Low-reynolds number wing response to an oscillating freestream with and without feed forward control," AIAA Paper 2009-143, 2009.

${ }^{29}$ Williams, D., Kerstens, W., Pfeiffer, J., King, R., and Colonius, T., "Unsteady lift suppression with a robust closed loop controller," Active Flow Control II, Springer-Verlag, Berlin, 2010, pp. 19-30.

${ }^{30}$ Kerstens, W., Williams, D., Pfeiffer, J., King, R., and Colonius, T., "Closed loop control of a wing's lift for 'gust' suppression," AIAA Paper 2010-4969, 2010.

${ }^{31}$ Gursul, I. and Ho, C.-M., "High aerodynamic loads on an airfoil submerged in an unsteady stream," AIAA Journal, Vol. 30, No. 4, 1992, pp. 1117-1119.

${ }^{32}$ Gursul, I., Lin, H., and Ho, C.-M., "Parametric effects on lift force of an airfoil in unsteady freestream," AIAA Journal, Vol. 34, No. 5, 1996, pp. 1085-1087.

${ }^{33}$ Soltani, M. R., Bragg, M. B., and Brandon, J. M., "Measurements on an oscillating 70-deg delta wing in subsonic flow," Journal of Aircraft, Vol. 27, No. 3, 1990, pp. 211-217.

${ }^{34}$ Gursul, I. and Ho, C.-M., "Vortex breakdown over delta wings in unsteady freestream," AIAA Journal, Vol. 32, No. 2, 1994, pp. 433-436.

${ }^{35}$ Shi, Z.-W. and Ming, X., "Effects of unsteady freestream on aerodynamic characteristics of pitching delta wing," Journal of Aircraft, Vol. 45, No. 6, 2008, pp. 2182-2185.

${ }^{36}$ Heron, I. and Myose, R. Y., "Delta wing vortex-burst behavior under a dynamic freestream," Journal of Aircraft, Vol. 46, No. 5, 2009, pp. 1500-1512.

${ }^{37}$ Pierce, G. A., Kunz, D. L., and Malone, J. B., "The effect of varying freestream velocity on airfoil dynamic stall characteristics," Journal of the American Helicopter Society, Vol. 23, No. 2, 1978, pp. 27-33.

${ }^{38}$ Leishman, J. G., "Challenges in modelling the unsteady aerodynamics of wind turbines," AIAA Paper 2002-0037, 2002.

${ }^{39}$ Favier, D., Rebont, J., and Maresca, C., "Large-amplitude fluctuations of velocity and incidence of an oscillating airfoil," AIAA Journal, Vol. 17, No. 11, 1979, pp. 1265-1267. 
${ }^{40}$ Favier, D., Agnes, A., Barbi, C., and Maresca, C., "Combined translation/pitch motion-A new airfoil dynamic stall simulation," Journal of Aircraft, Vol. 25, No. 9, 1988, pp. 805-814.

${ }^{41}$ Granlund, K., Monnier, B., Ol, M., and Williams, D., "Airfoil longitudinal gust response in separated vs. attached flows," Physics of Fluids, Vol. 26, No. 2, 2014, pp. 1-14.

${ }^{42}$ McCroskey, W. J. and Philippe, J. J., "Unsteady viscous flow on oscillating airfoils," AIAA Journal, Vol. 13, No. 1, 1975, pp. $71-79$.

${ }^{43}$ McCroskey, W. J., Carr, L. W., and McAlister, K. W., "Dynamic stall experiments on oscillating airfoils," AIAA Journal, Vol. 14, No. 1, 1976, pp. 57-63.

${ }^{44}$ Martin, J. M., Empey, R. W., McCroskey, W. J., and Caradonna, F. X., "An experimental analysis of dynamic stall on an oscillating airfoil," Journal of the American Helicopter Society, Vol. 19, No. 1, 1974, pp. 26-32.

${ }^{45}$ Ham, N., Bauer, P., and Lawrence, T., "Wind tunnel generation of sinusoidal lateral and longitudinal gusts by circulation of twin parallel airfoils," NASA STI/Recon Technical Report N, Vol. 75, 1974, pp. 29-51.

${ }^{46}$ Retelle, J. P., McMichael, J. M., and Kennedy, D. A., "Harmonic optimization of a periodic flow wind tunnel," Journal of Aircraft, Vol. 18, No. 8, 1981, pp. 618-623.

${ }^{47}$ Szumowski, A. and Meier, G., "Forced oscillations of airfoil flows," Experiments in Fluids, Vol. 21, No. 6, 1996, pp. 457464.

${ }^{48}$ Gompertz, K., Jensen, C., Kumar, P., Peng, D., Gregory, J. W., and Bons, J. P., "Modification of transonic blowdown wind tunnel to produce oscillating freestream Mach number," AIAA Journal, Vol. 49, No. 11, 2011, pp. 2555-2563.

${ }^{49}$ Goodrich, M. and Gorham, J., "Wind tunnels of the western hemisphere," Federal Research Division Library of Congress, Washington, DC, 2008, pp. 81-82.

${ }^{50}$ Furman, Y., Müller-Vahl, H., and Greenblatt, D., "Development of a low-speed oscillatory-flow wind tunnel," AIAA Paper 2013-0505, 2013.

${ }^{51}$ Müller-Vahl, H., Strangfeld, C., Nayeri, C. N., Paschereit, C. O., and Greenblatt, D., "Control of thick airfoil, deep dynamic stall using steady blowing," AIAA Journal, Vol. 53, No. 2, 2015, pp. 277-295.

${ }^{52}$ Goett, H. J. and Bullivant, W. K., "Tests of NACA 0009, 0012, and 0018 airfoils in the full-scale tunnel," NACA Report No. 647,1938

${ }^{53}$ Abbott, I. H., Theory of wing sections, including a summary of airfoil data, Courier Corporation, 1959

${ }^{54}$ Jacobs, E. N. and Sherman, A., "Airfoil section characteristics as affected by variations of the Reynolds number," NACA Technical Report No. 586, 1937.

${ }^{55}$ Raghunathan, S. and Ombaka, O. O., "A thick symmetrical aerofoil oscillating about zero incidence angle," International Journal of Heat and Fluid Flow, Vol. 7, No. 2, 1986, pp. 155-159.

${ }^{56}$ Timmer, W. A., "Two-dimensional low-Reynolds number wind tunnel results for airfoil NACA 0018," Wind Engineering, Vol. 32, No. 6, 2008, pp. 525-537.

${ }^{57}$ Gerakopulos, R., Boutilier, M. S., and Yarusevych, S., "Aerodynamic characterization of a NACA 0018 airfoil at low Reynolds numbers," AIAA Paper 2010-4629, 2010.

${ }^{58}$ Wickens, R. H., "Wind tunnel investigation of dynamic stall of a NACA 0018 airfoil oscillating in pitch," Tech. Rep. NAE-AN-27, National Research Council Canada, 1985.

${ }^{59}$ Sheng, W., Galbraith, R. A., and Coton, F. N., "Improved dynamic-stall-onset criterion at low Mach numbers," Journal of Aircraft, Vol. 44, No. 3, 2007, pp. 1049-1052.

${ }^{60}$ Sheng, W., McD. Galbraith, R. A., and Coton, F. N., "Return from aerofoil stall during ramp-down pitching motions," Journal of Aircraft, Vol. 44, No. 6, 2007, pp. 1856-1864.

${ }^{61}$ Krist, S. L., Biedron, R. T., and Rumsey, C. L., "CFL3D user's manual (version 5.0)," NASA/TM-1998-208444, 1998.

${ }^{62}$ Spalart, P. R. and Allmaras, S. R., "A one-equation turbulence model for aerodynamic flows," Recherche Aerospatiale, Vol. 1, 1994, pp. 5-21.

${ }^{63}$ Menter, F. R., "Two-equation eddy-viscosity turbulence models for engineering applications," AIAA Journal, Vol. 32 , No. 8, 1994, pp. 1598-1605.

${ }^{64}$ Cécora, R.-D., Radespiel, R., Eisfeld, B., and Probst, A., "Differential Reynolds-stress modeling for aeronautics," AIAA Journal, Vol. 53, No. 3, 2015, pp. 739-755.

${ }^{65}$ Rumsey, C. L., "Reynolds-averaged Navier-Stokes analysis of zero efflux flow control over a hump model," Journal of Aircraft, Vol. 44, No. 2, 2007, pp. 444-452.

${ }^{66}$ Strangfeld, C., Müller-Vahl, H., Greenblatt, D., Nayeri, C. N., and Paschereit, C. O., "Airfoil subjected to high-amplitude free-stream oscillations: theory and experiments," AIAA Paper 2014-2926, 2014.

${ }^{67}$ Rumsey, C. L., Lee-Rausch, E. M., and Watson, R. D., "Three-dimensional effects in multi-element high lift computations," Computers 86 Fluids, Vol. 32, No. 5, 2003, pp. 631-657.

${ }^{68}$ McCroskey, W. J., "Unsteady airfoils," Annual Review of Fluid Mechanics, Vol. 14, No. 1, 1982, pp. $285-311$. 